\title{
Centrifugal Assay for Fluorescence-Based Cell Adhesion Adapted to the Analysis of Ex Vivo Cells and Capable of Determining Relative Binding Strengths
}

\author{
Emiliana Giacomello ${ }^{1}$, Johanna Neumayer ${ }^{2}$, Alfonso Colombatti ${ }^{1,3}$ and Roberto \\ Perris ${ }^{1,4}$ \\ ${ }^{1}$ Centro di Riferimento Oncologico Aviano, Italy; ${ }^{2 T E C A N ~ A u s t r i a, ~ S a l z b u r g, ~ A u s t r i a ; ~}$ \\ ${ }^{3}$ University of Udine, Udine; and ${ }^{4}$ University of Parma, Parma, Italy
}

BioTechniques 26:758-766 (April 1999)

\begin{abstract}
Cell adhesion assays are widely used to identify novel cellular ligands, novel cell surface receptors for these ligands and to elucidate the mechanisms responsible for the underlying cellular and molecular interactions. We report here the development of a novel centrifugal assay for fluorescence-based cell adhesion (CAFCA) that offers a number of advantages over the currently available assays. CAFCA is based on two centrifugation steps: one to allow for the synchronization of the initial cell-substratum contact and one to enable both a defined removal force to be exerted onto the cells for displacement of unbound cells and determination of the relative binding strengths of adhering cells. The fluorescently tagged cells are monitored in specifically devised, disposable microplate assemblies by a two-sided fluorescence detection through the computer-interfaced SPECTRAFLUOR microplate fluorometer. The assay is rapid, accurate, reproducible and adaptable to small numbers of delicate primary cells that can ideally be labeled with the fluorochrome calcein AM (or analogous vital fluorescent dyes). Most uniquely, CAFCA provides (i) means of assessing the precise number of cells bound to a given substratum out of the total amount of cells contained within the population to be analyzed and (ii) a means of establishing the attachment strengths (i.e., dynes/cell) in a high number of samples/conditions simultaneously. CAFCA is therefore expected to make a substantial methodological and conceptual contribution to the range of available assays aimed at examining cellular interactions in vitro and promises the potential of being able to transpose automated versions of these tests for routine use in laboratories.
\end{abstract}

\section{INTRODUCTION}

Interplays between cells and their surrounding extracellular matrix (ECM) are thought to be crucially important during embryonic development, the maintanance of a healthy individual and the aberrant trafficking of transformed malignant cells. The assessment of cell-substratum interactions under static conditions has traditionally relied upon the use of cell adhesion assays involving radioactive incorporation, manual counting of selected microscopic fields or various colorimetric protocols to determine the number of cells bound to a given cellular or molecular substratum (2). Recent non-isotopic assay procedures based on cell labeling with various fluorescent compounds $(1,3,4,9,13)$ have eliminated the toxicity aspect, but the majority of these assays are still based upon the removal of unbound cells by arbitrarily performed washings (even if they are accomplished by automated systems). Exceptions are the recently described procedures in which nonadherent cells are removed from the substratum by either buoyancy (12) or the more elaborate ones involving well-controlled hydrodynamic forces as removal forces (5). It is evident that the routine cell adhesion assays adopted thus far might not yield ideal results when applied to high-throughput screening of ex vivo cells, and a more stringent evaluation of their traits reveals that they suffer from some serious limitations even when they are applied to cell lines. Since in all these assays the unbound cells are discarded, an accurate estimation of the percentage cells, out of the total number of cells analyzed, cannot be accomplished. Even more troublesome is 
the inability of these assays to synchronize the initial cell-substratum binding events and to apply defined and reproducible gravitational forces to the attached and nonattached cells. The purpose of the latter is to assure the dislodging of cells above a given threshold of binding strength, without exposing cells to tensional stress derived from shear forces (as in the case of many hydrodynamic-based assays); and to afford important information about the tenacity of the cell-cell/cell-substratum interaction. A cell-adhesion assay aimed at circumventing these limitations was first introduced by Lotz and McClay et al. $(8,10)$ and was further exploited by us $(7,11,14)$ to emphasize its wide application potential. Nonetheless, substantial drawbacks hamper use of the method in research laboratories worldwide. These drawbacks include (i) the high costs and health risks involved with the use of radioisotopes, (ii) the laborious and time-consuming nature of the procedure, (iii) the impracticality of analyzing a large number of samples and $(i v)$ the difficulty in applying the assay to cells sampled from healthy and diseased individuals. These restrictions have led us to develop a novel Centrifugal Assay for Fluorescencebased Cell Adhesion (CAFCA). The application potential of this assay for the quantitative analysis of cell-cell and cellECM adhesion in ex vivo cells is demonstrated here using as model cells B lymphocytes obtained from individuals affected by chronic-lymphocytic leukemia (CLL-B) and primary and established endothelial cell lines.

\section{MATERIALS AND METHODS}

\section{Cells}

Primary endothelial cells from human umbilical cords (Boehringer Ingelheim BioWhittaker, Verviers, Belgium) and the murine endothelioma cell line Hend 80 (kindly received from Dr. Federico Bussolino, University of Torino, Italy) were grown according to standard protocols and assayed with and without prior activation by treatment with $10-100 \mu \mathrm{g} / \mathrm{mL}$ of lipopolysaccharide (LPS) or $10 \mathrm{ng} / \mathrm{mL}$ of tumor necrosis factor alpha $(\mathrm{TNF} \alpha)$ for $16 \mathrm{~h}$. Peripheral B lymphocytes were separated according to standard procedures on Ficoll ${ }^{\circledR}$ gradients from heparinized whole blood obtained after informed consent from randomly selected CLL patients that were diagnosed and undergoing treatment at the Department of Medical Oncology, Centro di Riferimento Oncologico.

\section{Preparation of Adhesion Substrata}

For optimal performance of the assay, we have designed disposable 6-well microwell plates made up of flexible polyvinyl chloride (PVC) and denoted CAFCA miniplates (Figure 1A). These are devised as a bottom and a top miniplate (Figure 1A) and are identical, with the exception that the upper surface of the bottom one is covered with a strongly adhesive tape (protected by a peel-off paper). Both units are provided with two 0.2 -mm-wide/0.1-mm-deep grooves that extend parallel on each side of the wells and measure $57-\mathrm{mm}$ long. These lateral grooves serve as collecting ducts for the residual medium upon miniplate assembly (Figure 1, A and D). Wells of the bottom miniplate are the ones intended to be coated with the ligand molecule, cell monolayer or analogous substratum to be tested. In cases where exceptionally large cells are assayed at low centrifugal forces, it may be conve- 
nient to coat the wells of the top miniplate with $0.1 \%$ poly-Llysine to achieve maximal retention of the dislodged cells in the wells of the top CAFCA miniplate. The latter is essential for being able to precisely determine the ratio of bound vs. unbound cells (see below). For the experiments described, bottom wells were coated overnight at $4{ }^{\circ} \mathrm{C}$ with the following purified ECM molecules dissolved in $10 \mu \mathrm{g} / \mathrm{mL}$ of $0.05 \mathrm{M}$ bicarbonate buffer, $\mathrm{pH} 9.6$, containing human plasma fibronectin, tenascin- $\mathrm{C}$ and a complex of laminins at 1:10:11 (respective chain compositions: $\alpha 1 \beta 1 \gamma 1, \alpha 5 \beta 1 \gamma 1$ and $\alpha 5 \beta 2 \gamma 1$ ), collagen type I, tetrameric collagen type IV, tetrameric collagen type VI, thrombospondin-1, von Willebrand Factor (vWF) and fibrinogen. Uncovered surface areas of the wells were blocked with $0.5 \%$ Tween ${ }^{\circledR} 20$ in the same buffer for $\geq 60 \mathrm{~min}$ at room temperature, followed by extensive washing. In cases where the interaction of lymphocytes with the endothelium was examined, HUVEC or Hend 80 cells were plated at a density of 15-20000 cells per well and pre-grown in Dulbecco's modified Eagle medium (DMEM) with $10 \%$ fetal calf serum (FCS) for up to $16 \mathrm{~h}$ to reach con-

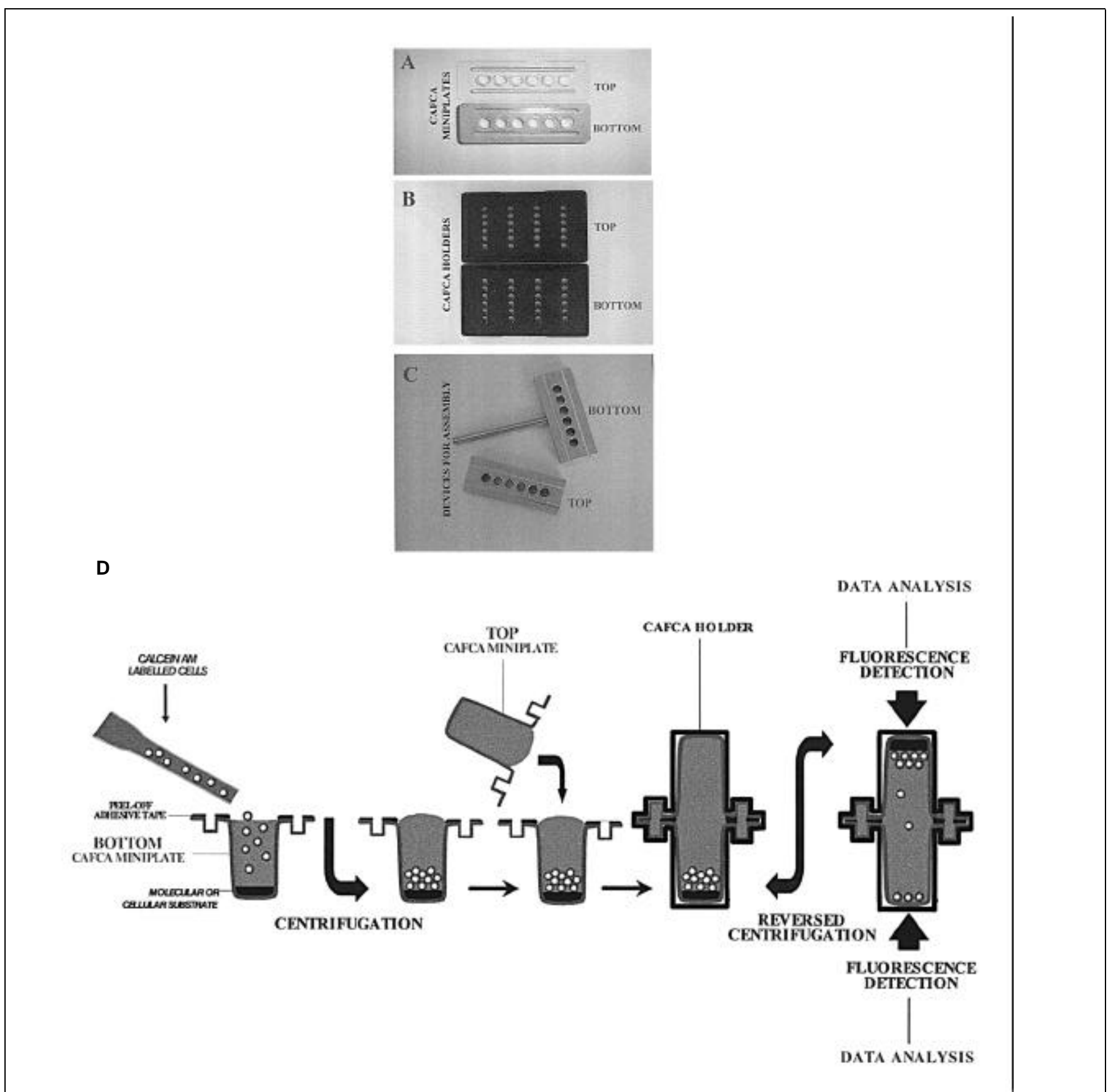

Figure 1. Specific accessories devised for CAFCA. (A) Disposable 6-well PVC CAFCA miniplates. These are designed as a bottom miniplate, covered with a strongly adhesive tape protected by peel-off paper, and intended to be coated with the molecular or cellular substratum to be analyzed, and an uncoated top miniplate with no tape. (B) Black, hard-plastic CAFCA holders, serving as supports for the CAFCA miniplates during the centrifugation steps and allowing for maximal shielding of the inter-well "spill-off" during fluorescence readings in the microplate fluorometer. These holders are similarly devised as a top and a bottom unit and are assembled by pressure-snapping. (C) Light-metal devices to be used for assuring optimal affixing of the top and bottom CAFCA miniplate units during assembly. All these accessories are part of a "CAFCA kit" that is commercially available from TECAN Austria. This kit can optionally also include a semi-hydraulic vise to be used for the maximal pressuring of the CAFCA miniplates during assembly. (D) Schematic diagram outlining the main steps of the CAFCA procedure. 
fluence before the assay. To allow for rapid attachment and spreading of the endothelial cells, bottom wells were precoated overnight with $10 \mu \mathrm{g} / \mathrm{mL}$ of vWF, which was independently ascertained to be nonpermissive for lymphocyte adhesion.

\section{Assay Procedure}

The procedure for CAFCA is schematically depicted in Figure 1D. Lymphocytes were labeled with $2 \mu \mathrm{M}$ Calcein AM (Molecular Probes, Eugene, OR, USA) for 5-15 min at $37^{\circ} \mathrm{C}$ in phosphate-buffered saline (PBS) followed by extensive washing and then aliquoted at 100-30000 cells/well in the bottom miniplates containing the substratum molecule, or an endothelial cell monolayer, and RPMI medium supplemented with $0.1 \%$ polyvinylpyrrolidone (PVP, $M_{\mathrm{r}} 360000$; Sigma-Aldrich GmbH, Harz, Germany) or whole human plasma obtained from healthy donors after informed consent. Since the fluorescent signal emitted by the labeled cells is transferred through the whole CAFCA miniplate assembly (Figure 1D), it was necessary to identify a soluble nontoxic "fluorescence quencher" to prevent interference from the sig- nal emanated from the counterposing well of the assembly. Comparison of a number of candidate agents led to the identification of India ink, at a $2 \%$ concentration, as the most efficient one and parallel tests ascertained that incubation for up to $4 \mathrm{~h}$ in medium supplemented with these concentrations of India ink did not affect the survival or proliferation rate of the cells. Direct comparisons of the extent of binding a number of both $\mathrm{T}$ and $\mathrm{B}$ lymphocyte lines to fibronectin using a previously described radioactive procedure $(11,14)$ also indicated that the presence of India ink had no effect on the adhesion behavior of the cells. Bottom miniplates were assembled with black hard plastic holders (CAFCA holders) (Figure 1B; TECAN Austria GmbH, Grodig, Austria) that are specifically devised as supports during centrifugation and fluorescence detection. These units were centrifuged either at $11 \times g$ or $170 \times g$ for studies on lymphocyte-endothelium or lymphocyte-ECM interactions, respectively. Thereafter, bottom and top miniplates were mounted together using the light-metal devices shown in Figure 1C, and the outermost bottom and top portions of the black CAFCA holders were similarly snapped together. Miniplate assemblies contained within the

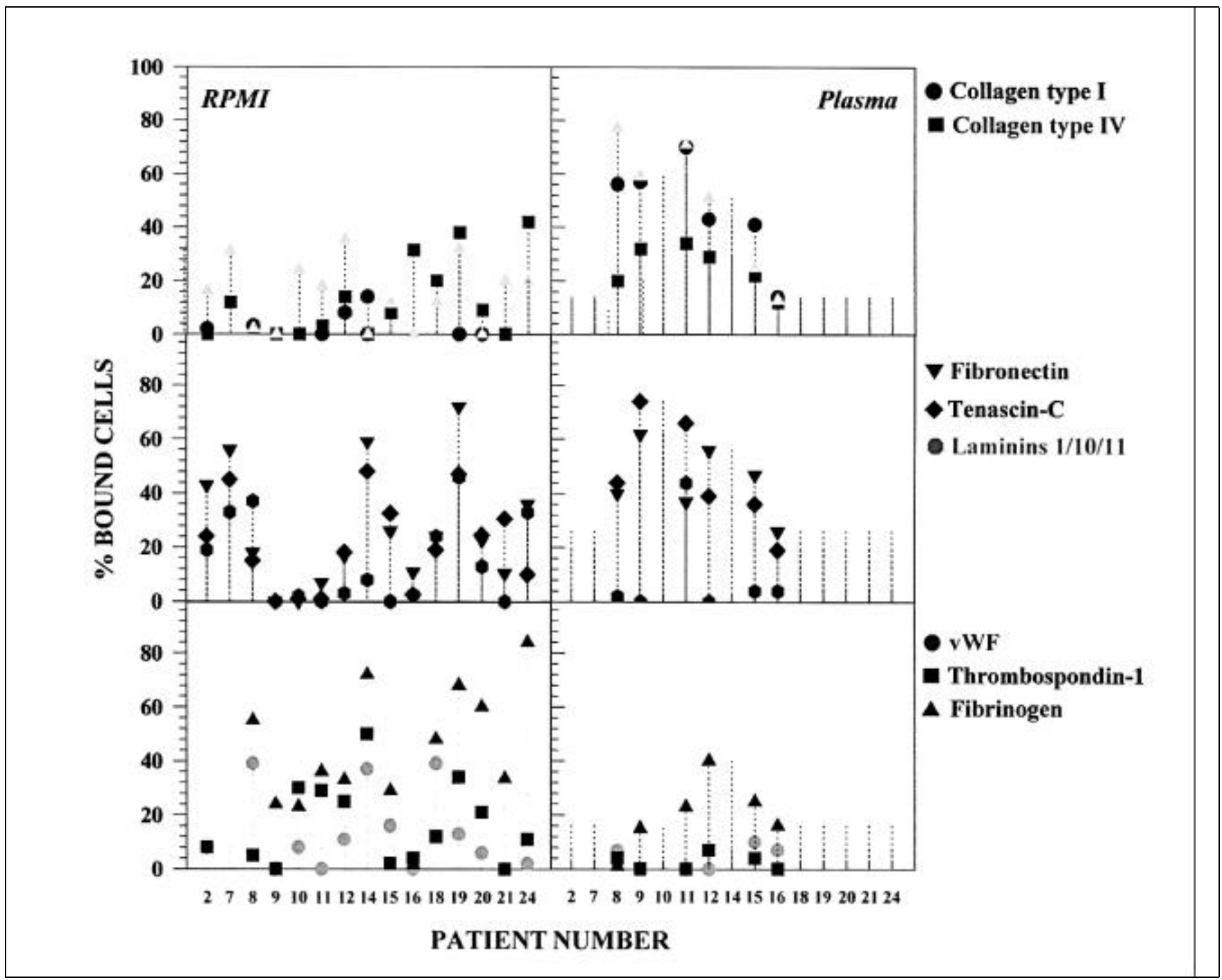

Figure 2. Patterns of ECM adhesion of B lymphocytes from 15 randomly selected CLL-B patients. Results were established by CAFCA using either the cell-culture medium RPMI containing 0.1\% PVP or human plasma from healthy donors. Centrifugation forces were $170 \times g$ in the first step and $43 \times g$ in the second reverse step. Standard deviation from quadruplicate tests was $<20 \%$. 
holders were then reversed-centrifuged at various forces ranging from $11-1065 \times g(1065 \times g$ is the maximum force that can be used without causing overt cell damage) for $5 \mathrm{~min}$ at $37^{\circ} \mathrm{C}$ in a digital thermally regulated centrifuge equipped with a 4armed Model CR422 Swinging Bucket Rotor (Jouan SA, St. Herblain, France) capable of accommodating up to 4 standard-sized microwell plates simultaneously. Fluorescence contained by the top (non-bound cells) and bottom (bound cells) wells was measured with the SPECTRAFLOUR Computer-Interfaced Microplate Fluorometer (TECAN Austria $\mathrm{GmbH}$ ) using a 485-/535-nm (excitation/emission) filter set. This fluorometer is specifically devised to allow for detection of the emitted fluorescence from two measurement directions (i.e., both top and bottom sides of the microplate) without need for mechanical switching of the optical unit. Fluorescence data were automatically imported into an Excel ${ }^{\circledR}$-based CAFCA software program for calculating the percentage of bound cells according to the following equation:

bottom fluorescence/(bottom + top fluorescence) $\times 100$

[Eq. 1]

When there is a specific interest in estimating the actual number of substratum bound cells vs. the total number introduced into the system, a parallel calibration curve could be generated with equivalently labeled cells. The cell number can then be extrapolated from these standard curves. Relative binding strengths expressed in dynes/cell were calculated as previously described $(8,14)$ according to Equation 2:

$$
\mathrm{A}_{d f}=\left(\mathrm{D}_{c}-\mathrm{D}_{m}\right) \times \mathrm{V}_{c} \times \mathrm{F}_{c}
$$

where $\mathrm{D}_{c}$ is the specific density of the cell (i.e., $1.07 \mathrm{~g} / \mathrm{cm}^{3}$; $\mathrm{D}_{m}$ is the specific density of the medium determined to range from $1012 \mathrm{~g} / \mathrm{cm}^{3}$ for the cell-adhesion medium with $0.5 \%$ PVP to $1.034 \mathrm{~g} / \mathrm{cm}^{3}$ for human plasma (averaged); $\mathrm{V}_{c}$ is the volume of the cell calculated on the average diameter of 9 $\mu \mathrm{m}$, which was estimated for the specific CLL-B cells examined here and $\mathrm{F}_{c}$ is the centrifugal force applied. The assessment of this parameter is analogous to that measured by Garcia et al., who used a spinning device to generate well-defined hydrodynamic forces to break the $\alpha 5 \beta 1$ integrin-fibronectin bonds in K562 cells (5).

\section{RESULTS AND DISCUSSION}

As a first goal in the development of CAFCA, we sought to identify a reliable procedure to fluorescently label cells by an efficient and preferably vital means. Comparative timeand dose-dependent dye incorporations, carried out in both established B- and T-cell lines (not shown) and the specific tumor B lymphocytes analyzed here, demonstrated that calcein AM yielded optimal cell labeling and the greatest intracellular retention. Another series of experiments was carried out with the intent to achieve maximal accuracy and reproducibility and to exploit the capacities of the assay. Naturally, the optimal assay conditions defined here are prone to adjustment in each individual case and according to an empirical determination of the specific parameters in the given experimental situation. A primary concern in our assay development was to establish the lower detection limits of the assay, such as to validate its applicability to the analysis of poorly represented cells, e.g., those that can be obtained from early developing embryos or biopsies/blood sampling in human beings. In the case of peripheral blood lymphocytes, the mini- mal number of cells that could accurately be detected with the present system configuration was as little as 200 cells per well, but this amount can readily be reduced to 100 cells per well if analyzing larger "anchorage-dependent-type" cells. Since freshly isolated CLL-B lymphocytes and many other primary and immortalized cell types might adhere in a "nonphysiological" manner to a number of commonly used blocking agents, we screened a variety of compounds and ascertained that a low percentage of Tween 20 was the optimal blocking agent.

CAFCA is based upon the application of defined (relative) centrifugal forces (to be calculated for the specific centrifuge used according to the conventional formula) in Equation 3:

$$
\mathrm{F}_{c}=1.12 \times r \times n^{2}
$$

where $r$ is the centrifugation radius in $\mathrm{mm}$, and $n$ is the centrifugation speed divided by 1000) to displace unbound cells from the substratum and differentially dislodge the adherent cells. Thereby, CAFCA offers the unique possibility of estimating the relative cell-cell and cell-substratum binding strengths in a large number of samples simultaneously, with no

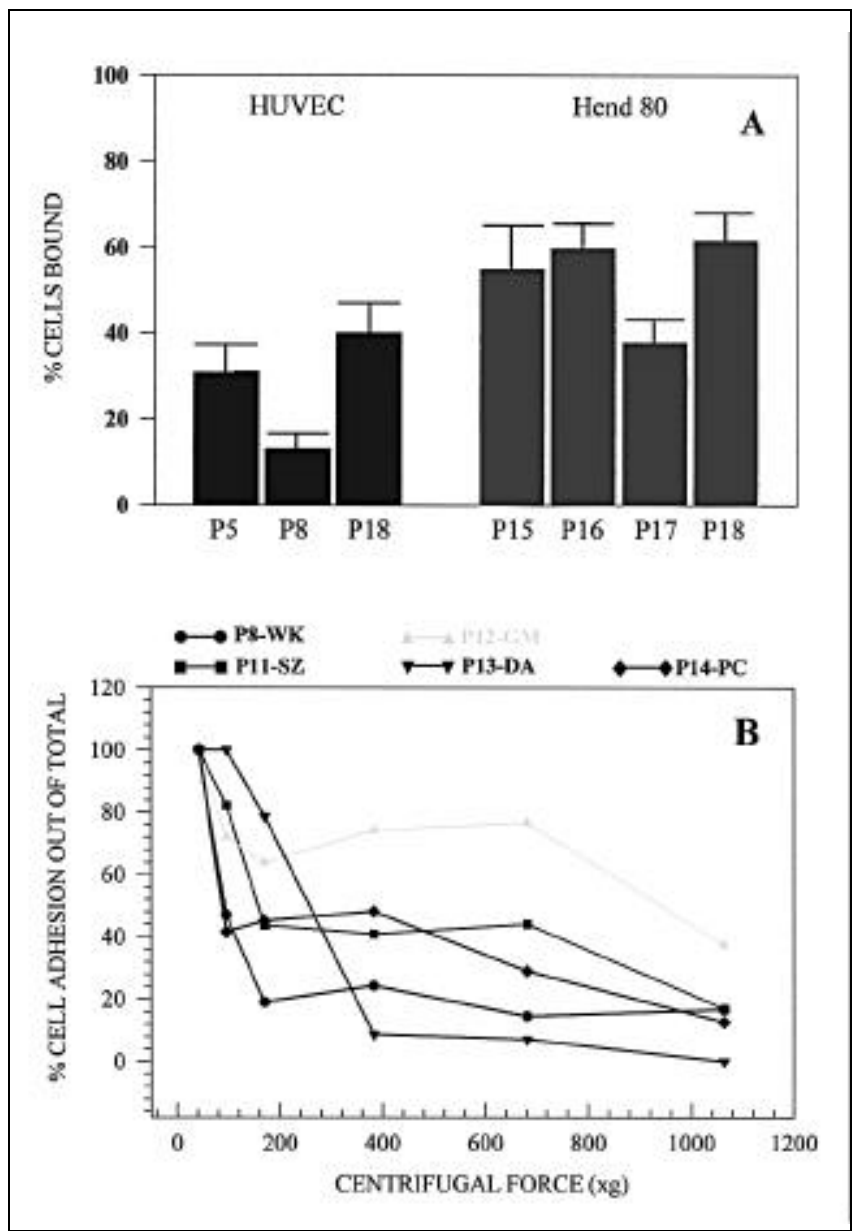

Figure 3. Adhesion of B lymphocytes. (A) Samples of B lymphocytes from 6 randomly selected CLL-B patients were assayed in RPMI medium for adhesion to two distinct endothelial cell types. Centrifugation forces were $11 \times g$ in the first step and $43 \times g$ in the second reverse-centrifugation step. HUVEC cells were grown on vWF substrata overnight and treated for $16 \mathrm{~h}$ with 10 $\mathrm{ng} / \mathrm{mL}$ of TNF $\alpha$. (B) Relative attachment strengths to fibrinogen in lymphocytes deriving from 5 of the CLL-B patients, when assayed in RPMI medium. Values represent the mean adhesion from triplicate tests when estimated as the percentage binding for each given force out of the maximal adhesion observed at the standard force of $43 \times \mathrm{g}$. Deviations varied from $7 \%-22 \%$. 
need for extra equipment. For this purpose, a medium with an intrinsic density assuring the correct balance between medium density and the specific mass of the cell to be analyzed should be used. Thus, if examining small suspension-growing cells, the intrinsic density of the medium should be lower than in cases where one analyzes larger anchorage-dependent cells. Moreover, we find that the previously proposed bovine serum albumin (BSA) and ovalbumin (7) medium additives can conveniently be replaced by the more inert high $M_{\mathrm{r}}$ PVP to yield the adequate medium density. Finally, the most crucial parameter to establish remains the optimal centrifugation force (Figure 1D) required to yield the lowest (i.e. <20\%) "nonphysiological binding" to the blocking agent, conventionally considered as nonspecific or background binding, while retaining maximal specific substratum attachment. In our experience, forces of $11 \times g$ (for cell-cell adhesion) and $170 \times g$ (for cell-substratum adhesion) in the first centrifugation step (Figure 1), and $\geq 43 \times g$ for the second reverse centrifugation step (for both types of interactions), can be adopted as the standards for work with cells of various sizes and types. We have empirically established that a 5-min centrifugation at the above indicated centrifugation forces, when using media with the indicated densities or human plasma, is sufficient to assure that all cells are brought in contact with the underlying substratum. In fact, according to Stoke's law:

$$
\mathrm{V}=2 \times \mathrm{g} \times\left[\mathrm{d}_{1}-\mathrm{d}_{2}\right] / 9 \times \eta
$$

where $d_{1}$ and $d_{2}$ are the relative densities of the cells and the media, and $\eta$ is the absolute viscosity coefficient of water at $20^{\circ} \mathrm{C}$ ), the maximal relative velocities $(\mathrm{V})$ with which cells would travel through the media would be $\leq 680 \mu$ per s, when exposed to a centrifugal force of $43 \times g$. This would imply that it would take $\leq 1 \mathrm{~min}$ for the cells to be displaced the approximately $2-\mathrm{cm}$ distance from the bottom to the top CAFCA miniplate unit during reverse-centrifugation. Increasing the duration of the second centrifugation step could be advantageous in some specific cases, e.g., comparing cells with apparently similar, high binding avidities to a specific substratum or substrata with strong adhesion-promoting capacities. In this case, increasing the centrifugation time at maximum applicable centrifugation force can disclose subtle differences in the relative binding strengths of the cells.

When examining cell-cell interactions, it could be important to identify a suitable growth substratum for the cells forming the "receiving" cell monolayer and ascertain the optimal status of this cell monolayer at the time of the assay, e.g., the endothelioma cell line Hend 80 can be used at a wide range of passages without any prior stimulation, whereas HUVEC invariably required pre-stimulation with cytokines (or analogous artificial compounds) and, in our hands, was unresponsive to lymphocyte interactions beyond 5 passages. Similarly, while most cell lines can efficiently attach and spread out on diverse types of plastics, primary and established endothelial cells generally have a more elaborate substratum requirement for optimal attachment/growth. Hence, a substratum that is nonpermissive for the adhesion of the spe- 
cific cell examined, while allowing formation of a confluent endothelial monolayer, has to be identified.

Peripheral B lymphocytes obtained from 24 randomly selected CLL-B patients exhibited a strongly diversified pattern of cell adhesion to these various matrix molecules (Figure 2). Cells bound relatively poorly to collagens, laminins, and thrombospondin-1, but more avidily to fibronectin, tenascin$\mathrm{C}$ and fibrinogen, when the assay was carried out in RPMI culture medium. This behavior was largely reversed if the substratum interaction was assayed in the presence of healthy human plasma (Figure 2), presumably due to the presence of adhesive components in the plasma, or as a result of a specific activation of the cells by circulating serum factors. Such possible cell activation, however, could not be reproduced by pre-treatment of the lymphocytes with phorbol esters (data not shown). Analysis of the CLL-B lymphocyte-endothelium interaction also revealed diverse binding capacities in cells deriving from different patients, with the levels of cell adhesion varying somewhat according to the endothelial cell type used (Figure 3A). Finally, one of the major advantages of CAFCA, namely the possibility to assess the relative binding strength of the cells to a given substratum, was exploited here on lymphocytes from a limited number of the patients examined. Exposure of cells attached to fibrinogen to incrementing centrifugal forces revealed that although a roughly comparable percentage of cells bound to this ECM substratum, their relative binding strengths varied markedly (Figure 3B). Thus, comparison of the relative attachment strengths of the cells at the extrapolated centrifugal force retaining $50 \%$ of the cells bound to fibrinogen revealed that (i) lymphocytes from patient P12-GM bound with an $A_{d f}=2.1 \times 10^{-4}$ dynes/cell, (ii) lymphocytes from patient P13-DA with a $A_{d f}=6.0 \times 10^{-5}$ and (iii) lymphocytes from patients P14-PC, P8-WK and P11-SZ with an $A_{d f}$ ranging from 2.0 to $3.6 \times 10^{-5}$.

While it is becoming increasingly more popular to examine the interaction of lymphocytes, as well as other normal and diseased cells, with the endothelium under "flow conditions" (i.e., such as to mimick the physiological shear stress encountered by circulating cells in blood vessels), static cell adhesion assays remain widely accepted for the quantitative and qualitative analysis of cellular and molecular adhesion phenomena. However, previously published cell-adhesion assays are encumbered by, among other problems, the difficulty in examining interactions of freshly sampled, diseased human cells in an accurate and reproducible manner. The cell-adhesion assay described herein affords an innovative way of analyzing many parameters and opens the potential to transpose these analyses to non-research laboratory settings. This is feasible due to the following properties of the assay: (i) the speed, which approximates $45 \mathrm{~min}$; (ii) the nonhazardous nature, since neither radioactive nor toxic substances are needed, and potentially infectious biological material can remain well-contained during the entire experiment; (iii) the lack of a need for particularly sophisticated and/or expensive equipment; (iv) the applicability to samples with relatively small numbers of cells; $(v)$ the unique possibility to simultaneously assess the number of fully vital cells that bind to a given substratum vs. the total number analyzed and (vi), most importantly, the possibility to concomittantly quantify the relative strengths with which these cells adhere to a molecular substratum or cell monolayer permitting evaluation of subtle differences in the adhesion patterns displayed by the cells. Additional potential advantages of CAFCA include the possibility of discriminating the binding events in subclasses of cells contained within a heterogeneous cell population, which is feasible by combining intracellular and extracellular cell type-specific labeling and use of specifically devoted fluorescent reagents to characterize some of the signal transduction phenomena involved in the adhesion process.

\section{ACKNOWLEDGMENTS}

We are indebted to a number of colleagues for providing purified ECM molecules and to Vittorina Zagonel, Simon Spazzapan and Mario Mazzuccato for both blood samples from CLL-B patients and human plasma from healthy donors. We also thank Cinzia Borghese and Maria Teresa Mucignat for their technical assistance. The work was in part supported by grants from AIRC (Associazione Italiana Ricerca sul Cancro) and Fondo Sanitario Nazionale.

\section{REFERENCES}

1.Akeson, A.L. and C.W. Woods. 1993. A fluorometric assay for the quantitation of cell adherence to endothelial cells. J. Immunol. Methods 163:181-185

2.Bongrard, P., P.M. Claesson and A.S.G. Curtis (Eds.). 1994. Studying Cell Adhesion. Springer-Verlag.

3.Braut-Boucher, F., J. Pichon, P. Rat, M. Adolphe, M. Aubery and J. Font. 1995. A non-isotopic, highly sensitive, fluorimetric, cell-cell adhesion microplate assay using calcein AM-labelled lymphocytes. J. Immunol. Methods 178:41-51.

4.De Clerck, L.S., C.H. Bridts, A.M. Mertens, M.M. Moens and W.J. Stevens. 1994. Use of fluorescent dyes in the determination of adherence of human leukocytes to endothelial cells and the effect of fluorochromes on cellular function. J. Immunol. Methods 172:115-124.

5.Garcia, A.J., F. Huber and D. Boettinger. 1998. Force required to break $\alpha 5 \beta 1$ integrin-fibronectin bonds in intact adherent cells is sensitive to integrin activation state. J. Biol. Chem. 273:10988-10993.

6.Goodwin, A.E. and B.U. Pauli. 1995. A new adhesion assay using buoyancy to remove non-adherent cells. J. Immunol. Methods 187:213-219.

7.Lallier, T., R. Deutzmann, R. Perris and M. Bronner-Fraser. 1994. Neural crest cell interactions with laminin: structural requirements and localization of the $\alpha 1 \beta 1$ binding site. Dev. Biol. 162:1031-1039.

8.Lotz, M.M, C.A. Burdsal, H.P. Erickson and D.R. McClay. 1989. Cell adhesion to fibronectin and tenascin: quantitative measurements of initial binding and subsequent strengthening response. J. Cell Biol. 109:17951805.

9.Maeda, Y., K. Tanaka, Y. Koga, X.-Y. Zhang, M. Sasaki, G. Kimura and K. Nomoto. 1993. A simple quantitative in vitro assay for thymocyte adhesion to thymic epithelial cells using a fluorescein diacetate. J. Immunol. Methods 157:117-123.

10.McClay, D.R., G.M. Wessel and R.B. Marchase. 1981. Intercellular recognition: quantitation of initial binding events. Proc. Natl. Acad. Sci. USA 78:4975-4979.

11.Segat, D., C. Pucillo, G. Marotta, R. Perris and A. Colombatti. 1994. Differential attachment of human neoplastic B cells to purified extracellular matrix molecules. Blood 83:1586-1594.

12.St. John, J.J., D.J. Schroen and H.T. Cheung. 1996. An adhesion assay using minimal shear force to remove nonadherent cells. J. Immunol. Methods 170:159-166.

13.van Kessel, K.P.M., T.C. Park and S.D. Wright. 1994. A fluorescence microassay for the quantitation of integrin-mediated adhesion of neutrophil. J. Immunol. Methods 172:25-31.

14.Yin, Z., E. Gabriele, A. Leprini, R. Perris and A. Colombatti. 1997. Differential cation regulation of the $\alpha 5 \beta 1$ integrin-mediated adhesion of leukemic cells to the central cell-binding domain of fibronectin. Cell Growth Differ. 8:1339-1347.

Address correspondence to Dr. Roberto Perris, Centro di Riferimento Oncologico, Division for Experimental Oncology 2, I-33081 Aviano (PN), Italy.Internet: rperris@ets.it 